International Journal of Applied Mathematics

Volume 34 No. 2 2021, 259-271

ISSN: $1311-1728$ (printed version); ISSN: 1314-8060 (on-line version)

doi: http://dx.doi.org/10.12732/ijam.v34i2.4

\title{
ON PERIODIC BOUNDARY VALUE PROBLEMS WITH AN OBLIQUE DERIVATIVE FOR A SECOND ORDER ELLIPTIC EQUATION
}

\author{
Maira Koshanova $^{1}$, Moldir Muratbekova ${ }^{2}$, \\ Batirkhan Turmetov ${ }^{3} \S$ \\ 1,2,3 Kh. Akhmet Yassawi International \\ Kazakh-Turkish University \\ B. Sattarhanov ave. 29 \\ Turkistan - 162200, KAZAKHSTAN
}

\begin{abstract}
In this paper, we study solvability of new classes of nonlocal boundary value problems for a second-order elliptic type equation. The considered problems are multidimensional analogues (in the case of circular domains) of classical periodic boundary value problems in rectangular domains.

To study the main problem, first, an auxiliary boundary value problem with inclined derivative is considered for the second order elliptic equation. The main problems are solved by reducing them to a sequential solution of the Dirichlet problem and the problem with inclined derivative. Theorems on the existence and uniqueness of a solution of considered problems are proved.
\end{abstract}

AMS Subject Classification: 35J15, 35J25

Key Words: elliptic equation; periodic problem; inclined derivative; boundary value problem; Dirichlet problem; solvability

\section{Introduction}

Let $\tilde{x}=\left(x_{1}, \ldots, x_{n-1}\right), \Omega_{m}=\left\{x \in R^{n}:|\tilde{x}|^{2}+\left|x_{n}\right|^{m}<1\right\}, n \geq 3, m>1, \partial \Omega_{m}=$ $\left\{x \in R^{n}:|\tilde{x}|^{2}+\left|x_{n}\right|^{m}=1\right\}$ be a boundary of the domain $\Omega_{m}$.

Received: December 15, $2021 \quad$ (C) 2021 Academic Publications

${ }^{\S}$ Correspondence author 
In $\Omega_{m}$ we consider an uniformly elliptic operator

$$
A(x, D)=\frac{\partial^{2}}{\partial x_{n}^{2}}+\sum_{p, q=1}^{n-1} a_{p q} \frac{\partial^{2}}{\partial x_{p} \partial x_{q}}+\sum_{j=1}^{n-1} b_{j} \frac{\partial}{\partial x_{j}}+c,
$$

where the coefficients $a_{p q}, b_{j}, c$ depend only on $\tilde{x}=\left(x_{1}, x_{2}, \ldots, x_{n-1}\right)$ and they are smooth enough, and $c \leq 0$.

For any point $x \in \Omega_{m}$ we put in conformity a point $x^{*}=\left(x_{1}, x_{2}, \ldots,-x_{n}\right)$. It is obvious that if $x \in \partial \Omega_{m}$, then $x^{*} \in \partial \Omega_{m}$.

Denote

$$
\begin{gathered}
\partial \Omega_{m}^{+}=\left\{x \in \partial \Omega_{m}: x_{n} \geq 0\right\}, \partial \Omega_{m}^{-}=\left\{x \in \partial \Omega_{m}: x_{n} \leq 0\right\}, \\
S=\left\{x \in \partial \Omega_{m}: x_{n}=0\right\} \equiv \partial \Omega_{m}^{+} \cap \partial \Omega_{m}^{-} .
\end{gathered}
$$

We introduce the operator $I_{*}[u](x)=\left.u(z)\right|_{z=x^{*}}$. Let a parameter $k$ take one of the values $k=1,2$. In $\Omega_{m}$ we consider the following problem:

$$
\begin{gathered}
A(x, D) u(x)=f(x), x \in \Omega, \\
u(x)+(-1)^{k} u\left(x^{*}\right)=g_{0}(x), x \in \partial \Omega_{+}, \\
\frac{\partial u(x)}{\partial x_{n}}+(-1)^{k} \frac{\partial u\left(x^{*}\right)}{\partial x_{n}}=g_{1}(x), x \in \partial \Omega_{+}, \\
u(x)=0, x \in S .
\end{gathered}
$$

Here $u\left(x^{*}\right)$ and $\frac{\partial u}{\partial x_{n}}\left(x^{*}\right)$ mean:

$$
u\left(x^{*}\right)=I_{*}[u](x), \frac{\partial u}{\partial x_{n}}\left(x^{*}\right)=I_{*}\left[\frac{\partial u}{\partial x_{n}}\right](x) .
$$

As a solution of problem (1)-(4) we call a function $u(x)$ from the class $C^{2}\left(\Omega_{m}\right) \cap C^{1}\left(\bar{\Omega}_{m}\right)$ which satisfies conditions (1)-(4) in the classical sense.

Problem (1)-(4) is an analogue of the periodic and antiperiodic problems for circular domains.

It should be noted that

$$
\frac{\partial u}{\partial x_{n}}\left(x^{*}\right)=I_{*}\left[\frac{\partial u}{\partial x_{n}}\right](x) \neq \frac{\partial}{\partial x_{n}} I_{*}[u](x),
$$

i.e. operator $I_{*}$ and differentiation operator $\frac{\partial}{\partial x_{n}}$ do not commute. Moreover, since

$$
\frac{\partial^{2} u}{\partial x_{n}^{2}}\left(x^{*}\right)=I_{*}\left[\frac{\partial^{2} u}{\partial x_{n}^{2}}\right](x)=\frac{\partial^{2}}{\partial x_{n}^{2}} I_{*}[u](x),
$$

then the following equality holds

$$
A(x, D) u\left(x^{*}\right) \equiv A(x, D) I_{*} u(x)=I_{*} A(x, D) u(x) .
$$


Further, if $x \in S$, then $x=(\tilde{x}, 0)$ and the corresponding points $x^{*}=$ $\left(x_{1}, x_{2}, \ldots, x_{n-1}, 0\right)$ also belong to the set $S$. Therefore, $u(x)$ belongs to the class $C^{1}\left(\bar{\Omega}_{m}\right)$, if the following condition holds:

$$
\begin{gathered}
g_{0}(\tilde{x}, 0)=u(\tilde{x}, 0)+(-1)^{k} u\left(\tilde{x}^{*}, 0\right) \\
=(-1)^{k}\left[u\left(\tilde{x}^{*}, 0\right)+(-1)^{k} u(\tilde{x}, 0)\right]=(-1)^{k} g_{0}\left(\tilde{x}^{*}, 0\right), \tilde{x} \in S .
\end{gathered}
$$

However, due to condition (4), the equality $u(\tilde{x}, 0)=0, \tilde{x} \in S$ holds, and therefore, the last condition can be rewritten in the form $g_{0}(\tilde{x}, 0)=g_{0}\left(\tilde{x}^{*}, 0\right)=$ $0, \tilde{x} \in S$, i.e.

$$
g_{0}(x)=0, x \in S .
$$

The following condition is also necessary

$$
\frac{\partial g_{0}}{\partial x_{j}}(x)-(-1)^{k} \frac{\partial g_{0}}{\partial x_{j}}\left(x^{*}\right)=0, x \in S, j=1,2, \ldots, n,
$$

and

$$
g_{1}(x)+(-1)^{k} g_{1}\left(x^{*}\right)=0, x \in S .
$$

Furthermore, we will assume that conditions (6) - (8) are satisfied. Note that similar problems for the Laplace and Poisson equations with normal derivatives of integer and fractional orders were studied in [1],[2],[3]. Moreover, in [4] similar problem was studied for a boundary operator with inclined derivative without degeneracy. We also note that degenerate boundary value problems with inclined derivative were studied in $[5],[6],[7],[8]$.

\section{Uniqueness of Solution}

We give the uniqueness theorem for the solution of problem (1)-(4).

Theorem 1. Let $k$ take one of the values $k=1,2$. If a solution of problem (1)-(4) exists, then it is unique.

Proof. Let $k=1$ and $u(x)$ be a solution of the homogenous problem (1)-(4). Then from condition (2) it follows that

$$
u(x)=u\left(x^{*}\right) \equiv I_{*}[u](x), x \in \partial \Omega_{m}^{+} .
$$


If $x \in \partial \Omega_{m}^{-}$, then it is obvious that $x^{*} \in \partial \Omega_{m}^{+}$. Therefore, from the boundary value condition (2) for the points $x \in \partial \Omega_{m}^{-}$we get

$$
u(x)=u\left(x^{*}\right) \equiv I_{*}[u](x), x \in \partial \Omega_{m}^{-} .
$$

Consequently, for all $x \in \partial \Omega_{m}$ we have

$$
u(x)-u\left(x^{*}\right)=0, x \in \partial \Omega_{m} .
$$

Denote $v(x)=u(x)-u\left(x^{*}\right)$. Then applying the operator $A(x, D)$ to $v(x)$, according to (5), we have

$$
A(x, D) v(x)=A(x, D) u(x)-A(x, D) u\left(x^{*}\right)=0, x \in \Omega_{m} .
$$

Hence, the function $v(x)$ is the solution of the following Dirichlet problem:

$$
A(x, D) v(x)=0, x \in \Omega_{m},\left.v(x)\right|_{\partial \Omega_{m}}=0 .
$$

Then, due to the uniqueness of the solution of the Dirichlet problem, $v(x)=$ $0, x \in \bar{\Omega}_{m}$. Therefore $u(x)=u\left(x^{*}\right) \equiv I_{*}[u](x), x \in \bar{\Omega}_{m}$. By the condition of the problem, $u(x)$ belongs to $C^{1}\left(\bar{\Omega}_{m}\right)$. Thus, from the condition $u(x)=u\left(x^{*}\right), x \in$ $\bar{\Omega}_{m}$, due to the equality:

$$
\begin{aligned}
& \frac{\partial}{\partial x_{n}} I_{*}[u](x)=\frac{\partial}{\partial x_{n}} u\left(x_{1}, \ldots, x_{n-1},-x_{n}\right) \\
= & -I_{*}\left[\frac{\partial u}{\partial x_{n}}\right]\left(x_{1}, \ldots, x_{n-1}, x_{n}\right) \equiv-\frac{\partial u\left(x^{*}\right)}{\partial x_{n}},
\end{aligned}
$$

we obtain

$$
\frac{\partial u}{\partial x_{n}}(x)=-\frac{\partial u}{\partial x_{n}}\left(x^{*}\right) \equiv-I_{*}\left[\frac{\partial u}{\partial x_{n}}\right](x), x \in \bar{\Omega}_{m} .
$$

On the other hand, by the boundary value condition (3), we have

$$
\frac{\partial u}{\partial x_{n}}(x)=\frac{\partial u}{\partial x_{n}}\left(x^{*}\right) \equiv I_{*}\left[\frac{\partial u}{\partial x_{n}}\right](x), x \in \partial \Omega_{m}^{+},
$$

and

$$
\frac{\partial u}{\partial x_{n}}(x)=\frac{\partial u}{\partial x_{n}}\left(x^{*}\right) \equiv I_{*}\left[\frac{\partial u}{\partial x_{n}}\right](x), x \in \partial \Omega_{m}^{-} .
$$

Consequently, for all $x \in \partial \Omega_{m}$ we have

$$
\frac{\partial u}{\partial x_{n}}(x)=\frac{\partial u}{\partial x_{n}}\left(x^{*}\right) \equiv I_{*}\left[\frac{\partial u}{\partial x_{n}}\right](x) .
$$


Then, adding the left-hand and right-hand sides of equalities (9) and (10), we obtain

$$
\frac{\partial u}{\partial x_{n}}(x)=0, x \in \partial \Omega_{m}
$$

Therefore, the solution of homogeneous problem (1)-(4) is also solution to the following problem

$$
A(x, D) u(x)=0, x \in \Omega_{m},\left.\frac{\partial u}{\partial x_{n}}(x)\right|_{\partial \Omega_{m}}=0,\left.u(x)\right|_{S}=0 .
$$

In [5] it is proved that the solution of the problem (11) is unique and therefore, $u(x) \equiv 0, x \in \bar{\Omega}_{m}$.

Let now $k=2$. In this case, if $u(x)$ is a solution of the homogenous problem (1)-(4), then from condition (2) for all point $x \in \partial \Omega_{m}^{+}$we get

$$
u(x)=-u\left(x^{*}\right), x \in \partial \Omega_{m}^{+} .
$$

In its turn, for points $\partial \Omega_{m}^{-}$:

$$
u(x)=-u\left(x^{*}\right), x \in \partial \Omega_{m}^{-} .
$$

Consequently, for all $x \in \partial \Omega_{m}$ we have

$$
u(x)+u\left(x^{*}\right)=0, x \in \partial \Omega_{m} .
$$

Further, if we denote $v(x)=u(x)+u\left(x^{*}\right)$, then the function $v(x)$ is a solution of the Dirichlet problem:

$$
A(x, D) v(x)=0, x \in \Omega_{m},\left.v(x)\right|_{\partial \Omega_{m}}=0,
$$

and due to the uniqueness of the solution of the Dirichlet problem, $v(x)=$ $0, x \in \bar{\Omega}_{m}$. Therefore, $u(x)=-u\left(x^{*}\right) \equiv-I_{*}[u](x)$,

$x \in \bar{\Omega}_{m}$. Then

$$
\frac{\partial u}{\partial x_{n}}(x)=-\frac{\partial u}{\partial x_{n}}\left(x^{*}\right) \equiv-\frac{\partial}{\partial x_{n}} I_{*}[u](x), \quad x \in \bar{\Omega}_{m} .
$$

Further, as in the case $k=1$ from the boundary value condition (3) we obtain:

$$
\begin{gathered}
\frac{\partial u}{\partial x_{n}}(x)=-\frac{\partial u}{\partial x_{n}}\left(x^{*}\right) \equiv-I_{*}\left[\frac{\partial}{\partial x_{n}} u\right](x), x \in \partial \Omega_{m}^{+}, \\
I_{*}\left[\frac{\partial}{\partial x_{n}} u\right](x) \equiv \frac{\partial u}{\partial x_{n}}\left(x^{*}\right)=-\frac{\partial u}{\partial x_{n}}(x), x \in \partial \Omega_{m}^{-}
\end{gathered}
$$


and consequently, we have

$$
\frac{\partial u}{\partial x_{n}}(x)=-\frac{\partial u}{\partial x_{n}}\left(x^{*}\right) \equiv-I_{*}\left[\frac{\partial}{\partial x_{n}} u\right](x)=\frac{\partial}{\partial x_{n}} I_{*}[u](x), x \in \partial \Omega_{m} .
$$

Hence

$$
\frac{\partial u}{\partial x_{n}}(x)=0, x \in \partial \Omega_{m} .
$$

In this case the function $u(x)$ satisfies the conditions of the problem (11). Then $u(x) \equiv 0, x \in \bar{\Omega}_{m}$.

\section{Existence of Solution}

In this section, we prove theorem on the existence and smoothness of a solution of the problem (1)-(4). Consider the following auxiliary problem

$$
A(x, D) z(x)=0, x \in \Omega_{m},\left.\frac{\partial z}{\partial x_{n}}(x)\right|_{\partial \Omega_{m}}=h(x),\left.z(x)\right|_{S}=0 .
$$

In [5] the following proposition is proved.

Lemma 2. Let $1-\frac{1}{m}<\lambda$, moreover let the number $\lambda+\frac{1}{m}$ be not integer. For any function $h(x) \in C^{\lambda}\left(\partial \Omega_{m}\right)$ a solution of problem (12) exists, is unique and belongs to the class $C^{\lambda+\frac{1}{m}}\left(\bar{\Omega}_{m}\right)$.

The following statement is true for the main problem.

Theorem 3. Let $k$ take one of the values $k=1,2$,

$$
1-\frac{1}{m}<\lambda<1, f(x) \in C^{\lambda}\left(\bar{\Omega}_{m}\right), g_{0}(x) \in C^{\lambda+1}\left(\partial \Omega_{m}^{+}\right), g_{1}(x) \in C^{\lambda}\left(\partial \Omega_{m}^{+}\right)
$$

and the matching conditions (6)-(8) be satisfied. Then a solution of the problem (1)-(4) exists, is unique and belongs to $C^{\lambda+\frac{1}{m}}\left(\bar{\Omega}_{m}\right)$.

Proof. Let $k=1$ and $u(x)$ be a solution of the problem (1)-(4). Consider the following function:

$$
v(x)=\frac{u(x)-u\left(x^{*}\right)}{2}, w(x)=\frac{u(x)+u\left(x^{*}\right)}{2} .
$$


Note that the functions $v(x)$ and $w(x)$ have the following properties:

$$
\begin{gathered}
v(x)=-v\left(x^{*}\right) \equiv-I_{*}[v](x) ; w(x)=w\left(x^{*}\right) \equiv I_{*}[w](x), \\
\frac{\partial v(x)}{\partial x_{n}}=-\frac{\partial}{\partial x_{n}} I_{*}[v](x)=I_{*}\left[\frac{\partial}{\partial x_{n}} v\right](x) \equiv \frac{\partial v\left(x^{*}\right)}{\partial x_{n}}, \\
\frac{\partial w(x)}{\partial x_{n}}=\frac{\partial}{\partial x_{n}} I_{*}[w](x)=-I_{*}\left[\frac{\partial}{\partial x_{n}} w\right](x) \equiv-\frac{\partial w\left(x^{*}\right)}{\partial x_{n}} .
\end{gathered}
$$

We find the conditions that the functions $v(x)$ and $w(x)$ will satisfy. Applying the operator $A(x, D)$ to the function $v(x)$, we have

$$
\begin{gathered}
A(x, D) v(x)=\frac{1}{2}\left[A(x, D) u(x)-A(x, D) u\left(x^{*}\right)\right] \\
=\frac{1}{2}\left[A(x, D) u(x)-I_{*} A(x, D) u(x)\right] \\
=\frac{1}{2}\left[f(x)-f\left(x^{*}\right)\right], x \in \Omega_{m} .
\end{gathered}
$$

Further, from boundary condition (2) it follows that

$$
\left.v(x)\right|_{\partial \Omega_{m}^{+}}=\left.\frac{1}{2}\left[u(x)-u\left(x^{*}\right)\right]\right|_{\partial \Omega_{m}^{+}}=\frac{1}{2} g_{0}(x) .
$$

If the point $x$ belongs to the lower part of the boundary, i.e. $x \in \partial \Omega_{m}^{-}$, then the corresponding point $x^{*}$ belongs to the upper part of the boundary, and therefore, again from the boundary condition (2), we obtain

$$
\begin{gathered}
\left.v(x)\right|_{\partial \Omega_{m}^{-}}=\left.\frac{1}{2}\left[u(x)-u\left(x^{*}\right)\right]\right|_{\partial \Omega_{m}^{-}}=-\left.\frac{1}{2}\left[u\left(x^{*}\right)-u(x)\right]\right|_{x^{*} \in \partial \Omega_{m}^{+}} \\
=-\frac{1}{2} g_{0}\left(x^{*}\right) .
\end{gathered}
$$

Moreover, condition (4) implies

$$
\left.v(x)\right|_{S}=\left.\frac{1}{2}\left[u(x)-u\left(x^{*}\right)\right]\right|_{S}=0 .
$$

Further, making similar actions with respect to $w(x)$ from equality (13), we obtain

$$
\begin{gathered}
A(x, D) w(x)=\frac{1}{2}\left[A(x, D) u(x)+A(x, D) u\left(x^{*}\right)\right] \\
=\frac{1}{2}\left[f(x)+f\left(x^{*}\right)\right], x \in \Omega_{m},
\end{gathered}
$$




$$
\begin{gathered}
\left.\frac{\partial w(x)}{\partial x_{n}}\right|_{\partial \Omega_{+}}=\left.\frac{1}{2}\left[\frac{\partial u(x)}{\partial x_{n}}+\frac{\partial}{\partial x_{n}} I_{*}[u](x)\right]\right|_{\partial \Omega_{+}}=\left.\frac{1}{2}\left[\frac{\partial u(x)}{\partial x_{n}}-\frac{\partial u}{\partial x_{n}}\left(x^{*}\right)\right]\right|_{\partial \Omega_{+}} \\
=\left.\frac{1}{2}\left[\frac{\partial u(x)}{\partial x_{n}}-I_{*}\left[\frac{\partial u}{\partial x_{n}}\right](x)\right]\right|_{\partial \Omega_{+}}=\frac{1}{2} g_{1}(x), x \in \partial \Omega_{m}^{+}, \\
\left.\frac{\partial w(x)}{\partial x_{n}}\right|_{\partial \Omega_{-}}=\left.\frac{1}{2}\left[\frac{\partial u(x)}{\partial x_{n}}+\frac{\partial}{\partial x_{n}} I_{*}[u](x)\right]\right|_{\partial \Omega_{-}}=\left.\frac{1}{2}\left[\frac{\partial u(x)}{\partial x_{n}}-\frac{\partial u\left(x^{*}\right)}{\partial x_{n}}\right]\right|_{\partial \Omega_{-}} \\
=\left.\frac{1}{2}\left[\frac{\partial u(x)}{\partial x_{n}}-I_{*}\left[\frac{\partial u}{\partial x_{n}}\right](x)\right]\right|_{\partial \Omega_{-}}=-\frac{1}{2} g_{1}\left(x^{*}\right), x \in \partial \Omega_{m}^{-} .
\end{gathered}
$$

Moreover, condition (4) implies

$$
\left.w(x)\right|_{S}=\left.\frac{1}{2}\left[u(x)+u\left(x^{*}\right)\right]\right|_{S}=0 .
$$

Denote,

$$
\begin{gathered}
f^{ \pm}(x)=\frac{1}{2}\left[f(x) \pm f\left(x^{*}\right)\right], 2 \tilde{g}_{0}(x)=\left\{\begin{array}{c}
g_{0}(x), x \in \partial \Omega_{m}^{+} \\
-g_{0}\left(x^{*}\right), x \in \partial \Omega_{m}^{-}
\end{array}\right. \\
2 \tilde{g}_{1}(x)=\left\{\begin{array}{c}
g_{1}(x), x \in \partial \Omega_{m}^{+} \\
-g_{1}\left(x^{*}\right), x \in \partial \Omega_{m}^{-}
\end{array}\right.
\end{gathered}
$$

Let us examine the smoothness of these functions. Let $f(x) \in C^{\lambda}\left(\bar{\Omega}_{m}\right)$. Then it is obvious that the functions $f^{ \pm}(x)$ also belong to the class $C^{\lambda}\left(\bar{\Omega}_{m}\right)$. Further, by the hypothesis of the theorem, the function $g_{0}(x)$ belongs to the class $C^{\lambda+1}\left(\partial \Omega_{m}^{+}\right)$and the matching conditions (6) and (7) are satisfied for it. Then the function $\tilde{g}_{0}(x)$ belongs to the class $C^{\lambda+1}\left(\partial \Omega_{m}\right)$. Similarly, the function $g_{1}(x)$ belongs to the class and the matching condition (8) is satisfied for it. Then the function $\tilde{g}_{1}(x)$ belongs to the class $C^{\lambda}\left(\partial \Omega_{m}\right)$. Thus, if $u(x)$ is a solution of problem (1)-(4), then the functions $v(x)$ and $w(x)$ satisfy the conditions of the following problems:

$$
A(x, D) v(x)=f^{-}(x), x \in \Omega,\left.v(x)\right|_{\partial \Omega}=\tilde{g}_{0}(x),
$$

with the additional condition

$$
\left.v(x)\right|_{S}=0
$$


and

$$
A(x, D) w(x)=f^{+}(x), x \in \Omega_{m},
$$

$$
\left.\frac{\partial w}{\partial x_{n}}(x)\right|_{\partial \Omega_{m}}=\tilde{g}_{1}(x),\left.w(x)\right|_{s}=0 .
$$

We study the problem (14). We will look for a solution to the problem in the form $v(x)=v_{1}(x)+v_{2}(x)$, where the functions $v_{1}(x)$ and $v_{2}(x)$ satisfy the conditions of the following problems

$$
\begin{aligned}
& A(x, D) v_{1}(x)=f^{-}(x), x \in \Omega,\left.v_{1}(x)\right|_{\partial \Omega}=0, \\
& A(x, D) v_{2}(x)=0, x \in \Omega,\left.v_{2}(x)\right|_{\partial \Omega}=\tilde{g}_{0}(x) .
\end{aligned}
$$

The problems (17) and (18) are classical Dirichlet problems, and for smooth data, solutions to these problems always exist. We need to clarify the smoothness of the solutions to these problems. It was proved in [9] that if $0<$ $\lambda<1, f^{-}(x) \in C^{\lambda}\left(\bar{\Omega}_{m}\right)$, then solution to the problem (17) belongs to the class $C^{\lambda+2}\left(\bar{\Omega}_{m}\right)$. The exact smoothness order of the solution to the problem (18) in the case $\tilde{g}_{0}(x) \in C^{\lambda+1}\left(\partial \Omega_{m}\right)$ is given in [5] and the order has the form $C^{\lambda+1}\left(\bar{\Omega}_{m}\right)$. In addition, due to the matching condition (6), the equality $\left.v_{2}(x)\right|_{S}=0$ holds. Thus, if $f^{-}(x) \in C^{\lambda}\left(\bar{\Omega}_{m}\right)$,

$\tilde{g}_{0}(x) \in C^{\lambda+1}\left(\partial \Omega_{m}\right)$, then the solution to the problem (14) exists and condition (15) holds for it. Further, we will look for a solution to the problem (16) in the form $w(x)=w_{1}(x)+w_{2}(x)$, where the functions $w_{1}(x)$ and $w_{2}(x)$ are solutions of the following problems:

$$
A(x, D) w_{1}(x)=f^{+}(x), \quad x \in \Omega_{m},\left.w_{1}(x)\right|_{\partial \Omega_{m}}=0,
$$

$$
\begin{aligned}
A(x, D) w_{2}(x)=0, \quad x \in & \Omega_{m} ; \\
& \left.\frac{\partial w_{2}(x)}{\partial x_{n}}\right|_{\partial \Omega_{m}}=\tilde{g}_{1}(x)-\frac{\partial w_{1}(x)}{\partial x_{n}},\left.w_{2}(x)\right|_{S}=0 .
\end{aligned}
$$

As we have already noticed, under the conditions of the theorem and the matching conditions (8), the function $f^{+}(x)$ belongs to the class $C^{\lambda}\left(\bar{\Omega}_{m}\right)$. Then the solution to the problem (19) exists, is unique and belongs to the class $C^{\lambda+2}\left(\bar{\Omega}_{m}\right)$. Consequently, $\frac{\partial w_{1}(x)}{\partial x_{n}} \in C^{\lambda+1}\left(\bar{\Omega}_{m}\right)$. Further, if $g_{1}(x) \in C^{\lambda}\left(\partial \Omega_{m}^{+}\right)$, then the function $\tilde{g}_{1}(x)-$ $\frac{\partial w_{1}(x)}{\partial x_{n}}$ at least belongs to the class $C^{\lambda}\left(\partial \Omega_{m}\right)$. 
With these data, according to Lemma 1, the solution to problem (20) exists, is unique, and belongs to the class $C^{\lambda+\frac{1}{m}}\left(\bar{\Omega}_{m}\right)$. Therefore, the solution to problem (16) exists, is unique and also belongs to the class $C^{\lambda+\frac{1}{m}}\left(\bar{\Omega}_{m}\right)$. Thus, we will the functions $v(x)$ and $w(x)$ from equalities (13). We show that if $v(x)$ and $w(x)$ are the solutions to problems (13) and (16), then the function $u(x)=v(x)+w(x)$ satisfies all the conditions of the problem (1)-(4). Indeed, applying the operator $A(x, D)$ to the function $u(x)=v(x)+w(x)$, we have

$$
\begin{gathered}
A(x, D) u(x)=A(x, D) v(x)+A(x, D) w(x) \\
=f^{+}(x)+f^{-}(x)=f(x), x \in \Omega .
\end{gathered}
$$

Further, according to the properties of the functions $v(x)$ and $w(x)$, we have

$$
\begin{aligned}
& u(x)-\left.u\left(x^{*}\right)\right|_{\partial \Omega_{m}^{+}}=v(x)+w(x)-\left.\left(v\left(x^{*}\right)+w\left(x^{*}\right)\right)\right|_{\partial \Omega_{m}^{+}} \\
= & v(x)-\left.v\left(x^{*}\right)\right|_{\partial \Omega_{m}^{+}}=\left.2 v(x)\right|_{\partial \Omega_{m}^{+}}=\left.2 \tilde{g}_{0}(x)\right|_{\partial \Omega_{m}^{+}}=g_{0}(x) .
\end{aligned}
$$

Similarly,

$$
\begin{gathered}
\frac{\partial u(x)}{\partial x_{n}}-\left.I_{*}\left[\frac{\partial u}{\partial x_{n}}\right](x)\right|_{\partial \Omega_{m}^{+}}=\frac{\partial v(x)}{\partial x_{n}}+\left.\frac{\partial w(x)}{\partial x_{n}}\right|_{\partial \Omega_{m}^{+}} \\
-I_{*}\left[\frac{\partial v}{\partial x_{n}}\right](x)-\left.I_{*}\left[\frac{\partial w}{\partial x_{n}}\right](x)\right|_{\partial \Omega_{m}^{+}}=\frac{\partial w(x)}{\partial x_{n}}-\left.I_{*}\left[\frac{\partial w}{\partial x_{n}}\right](x)\right|_{\partial \Omega_{m}^{+}} \\
=\left.2 \frac{\partial w(x)}{\partial x_{n}}\right|_{\partial \Omega_{m}^{+}}=\left.2 \tilde{g}_{0}(x)\right|_{\partial \Omega_{m}^{+}}=g_{1}(x) .
\end{gathered}
$$

Thus, the theorem is proved for the case $k=1$.

Let now, $k=2$ and $u(x)$ be a solution of problem (1)-(4) for this case. Applying the operator $A(x, D)$ to the function $v(x)$, as in the case $k=1$ from equality (13), we obtain

$$
\begin{gathered}
A(x, D) v(x)=\frac{1}{2}\left[A(x, D) u(x)-A(x, D) u\left(x^{*}\right)\right] \\
=\frac{1}{2}\left[f(x)-f\left(x^{*}\right)\right]=f^{-}(x), x \in \Omega_{m} .
\end{gathered}
$$

Further, from the boundary value condition (2) we obtain

$$
\begin{gathered}
\left.\frac{\partial v(x)}{\partial x_{n}}\right|_{\partial \Omega_{+}}=\left.\frac{1}{2}\left[\frac{\partial u(x)}{\partial x_{n}}-\frac{\partial}{\partial x_{n}} I_{*}[u](x)\right]\right|_{\partial \Omega_{+}} \\
=\left.\frac{1}{2}\left[\frac{\partial u(x)}{\partial x_{n}}+I_{*}\left[\frac{\partial u}{\partial x_{n}}\right](x)\right]\right|_{\partial \Omega_{+}}=\left.\frac{1}{2}\left[\frac{\partial u(x)}{\partial x_{n}}+\frac{\partial u}{\partial x_{n}}\left(x^{*}\right)\right]\right|_{\partial \Omega_{+}}
\end{gathered}
$$




$$
=\frac{1}{2} g_{1}(x), x \in \partial \Omega_{m}^{+} .
$$

Similarly, for $x \in \partial \Omega_{m}^{-}$we have

$$
\begin{gathered}
\left.\frac{\partial v(x)}{\partial x_{n}}\right|_{\partial \Omega_{m}^{-}}=\left.\frac{1}{2}\left[\frac{\partial u(x)}{\partial x_{n}}-\frac{\partial}{\partial x_{n}} I_{*}[u](x)\right]\right|_{\partial \Omega_{m}^{-}}=\left.\frac{1}{2}\left[\frac{\partial u(x)}{\partial x_{n}}+\frac{\partial u\left(x^{*}\right)}{\partial x_{n}}\right]\right|_{\partial \Omega_{m}^{-}} \\
=\left.\frac{1}{2}\left[\frac{\partial u\left(x^{*}\right)}{\partial x_{n}}+\frac{\partial u(x)}{\partial x_{n}}\right]\right|_{x^{*} \in \partial \Omega_{m}^{+}}=\frac{1}{2} g_{1}\left(x^{*}\right), x \in \partial \Omega_{m}^{-} .
\end{gathered}
$$

Moreover, condition (4) yields that

$$
\left.v(x)\right|_{S}=\left.\frac{1}{2}\left[u(x)-u\left(x^{*}\right)\right]\right|_{S}=0 .
$$

Further, for the function $w(x)$ from (13), we get

$$
\begin{gathered}
A(x, D) w(x)=\frac{1}{2}\left[A(x, D) u(x)+A(x, D) u\left(x^{*}\right)\right] \\
=\frac{1}{2}\left[f(x)+f\left(x^{*}\right)\right]=f^{+}(x), x \in \Omega_{m} \\
\left.w(x)\right|_{\partial \Omega_{+}}=\left.\frac{1}{2}\left[u(x)+u\left(x^{*}\right)\right]\right|_{\partial \Omega_{+}}=\frac{1}{2} g_{0}(x) \\
\left.w(x)\right|_{\partial \Omega_{m}^{-}}=\left.\frac{1}{2}\left[u(x)+u\left(x^{*}\right)\right]\right|_{\partial \Omega_{m}^{-}}=\left.\frac{1}{2}\left[u\left(x^{*}\right)+u(x)\right]\right|_{x^{*} \in \partial \Omega_{m}^{+}} \\
=\frac{1}{2} g_{0}\left(x^{*}\right) .
\end{gathered}
$$

Moreover, from (4) it follows that

$$
\left.w(x)\right|_{S}=\left.\frac{1}{2}\left[u(x)+u\left(x^{*}\right)\right]\right|_{S}=0 .
$$

We introduce the following functions

$$
2 \tilde{g}_{0}(x)=\left\{\begin{array}{c}
g_{0}(x), x \in \partial \Omega_{m}^{+} \\
g_{0}\left(x^{*}\right), x \in \partial \Omega_{m}^{-}
\end{array}, 2 \tilde{g}_{1}(x)=\left\{\begin{array}{c}
g_{1}(x), x \in \partial \Omega_{m}^{+} \\
g_{1}\left(x^{*}\right), x \in \partial \Omega_{m}^{-}
\end{array} .\right.\right.
$$

Thus, in this case for the functions $v(x)$ and $w(x)$ we obtain the following problems: 


$$
\begin{array}{ll}
A(x, D) v(x)=f^{-}(x), x \in \Omega ; & \left.\frac{\partial v(x)}{\partial x_{n}}\right|_{\partial \Omega_{m}}=\tilde{g}_{1}(x),\left.v(x)\right|_{S}=0, \\
A(x, D) w(x)=f^{+}(x), x \in \Omega_{m} ; & \left.w(x)\right|_{\partial \Omega_{m}}=\tilde{g}_{0}(x),\left.w(x)\right|_{s}=0 .
\end{array}
$$

The problems (21) and (22) are investigated in the same way as in the case $k=1$. Under the conditions of the theorem, the solutions to these problems exist, are unique and belong to the class $C^{\lambda+\frac{1}{m}}\left(\bar{\Omega}_{m}\right)$. Further, exactly as in the case $k=1$, it can be shown that the function $u(x)=v(x)+w(x)$ satisfies all the conditions of problem (1)-(4).

\section{Acknowledgements}

The work was supported by a grant from the Ministry of Science and Education of the Republic of Kazakhstan (Grant No. AP08855810).

\section{References}

[1] M.A. Sadybekov, B.Kh. Turmetov, On analogues of periodic boundary value problems for the Laplace operator in a ball, Eurasian Mathematical Journal, 3, No 1 (2012), 143-146.

[2] M.A. Sadybekov, B.Kh. Turmetov, On an analog of periodic boundary value problems for the Poisson equation in the disk, Differential equations, 50, No 2 (2014), 268-273; doi:10.1134/S0012266114020153.

[3] B.Kh. Turmetov, M.D.Koshanova, K.I. Usmanov, About solvability of some boundary value problems for Poisson equation in the ball conditions, Filomat, 32, No 3 (2018), 939-946.

[4] B. Turmetov, M. Koshanova, M. Muratbekova, On some analogues of periodic problems for Laplace equation with an oblique derivative under boundary conditions, e-Journal of Analysis and Applied Mathematics, 3 (2020), 13-27.

[5] Sh.A. Alimov, On a problem with an oblique derivative, Differ. Uravn., 17, No 10 (1981), 1738-17511. 
[6] Sh.A. Alimov, On a boundary value problem, Dokl. Akad. Nauk SSSR, 252, No 5 (1980), 1033-1034.

[7] D.I. Boyarkin, A boundary value problem with degeneration on the boundary along the manifold of codimension $k>2$, Zhurnal SVMO, 18, No 2 (2016), 7-10.

[8] P. Popivanov, Boundary value problems for the biharmonic operator in the unit ball, In: AIP Conf. Proc. 2159 (6th Intern. Conf. "New Trends in the Applications of Differential Equations in Sciences" (NTADES 2019), Bulgaria, July 1-4, 2019) (Ed. by: A. Slavova), Amer. Inst. Phys. (2019), $1-10$.

[9] D. Gilbarg, N. Trudinger, Elliptic Partial Differential Equations of Second Order, Springer-Verlag, Berlin (1977). 
